\title{
Avaliação da resposta sensório-motora e funcionalidade após a participação no Programa de Prevenção de Lesões FIFA "THE 11+"*
}

\author{
Response evaluation sensory-motor and \\ functionality after participation in \\ Program Injury Prevention FIFA "THE 11 +"
}

Vinícius Araújo Bispo' Márcio de Paula Oliveira ${ }^{2}$
Recebido em: 04/09/2014. Aprovado em: 10/09/2015.

1 Discente do Centro Universitário de Brasília UniCEUB, Brasil Brasília/Distrito Federal araujo_50@hotmail.com

2 Docente do Centro Universitário de Brasília UniCEUB, Brasil Brasília/Distrito Federal professor_marciooliveira@hotmail.com

\section{Resumo}

O futebol, devido ao alto nível de exigência dos atletas, está associado a diferentes tipos de lesões. A falta de programas preventivos contribuem para um aumento dessa ocorrência. $\mathrm{O}$ estudo proposto neste artigo avaliou a resposta sensório-motora e funcional de atletas de futebol de base de Brasília/DF, após realizarem o programa FIFA "The $11+$ ". Foram estudados 12 atletas do gênero masculino, com idade média de 14 anos, estatura $1,65 \mathrm{~cm}$ e peso de $52 \mathrm{~kg}$. Não houve grupo controle e interrupção dos treinos. A resposta sensório-motora foi feita por meio da eletromiografia nos músculos responsáveis pelo chute, vasto medial, lateral e reto femoral durante o Star Excursion Balance Test, e a funcionalidade foi feita por meio do Single Leg Hop Test em ambos os membros. Foi utilizado o teste T para amostras dependentes normais e o teste de Wilcoxon para amostras não normais. Os resultados demonstraram diferenças estatísticas entre as intervenções na EMG dos músculos vasto lateral $p=0,02$, reto femoral $\mathrm{p}=0,01$ e SLHT do membro inferior direito $\mathrm{p}=0,00$. Conclui-se que o programa FIFA “The 11+” promoveu aumento da resposta sensóriomotora e funcional no grupo de atletas estudados.

Palavras-chave: Eletromiografia. FIFA “The 11+”. Prevenção.

\begin{abstract}
Football, due to the high standards of the athletes is associated with different types of injuries. The lack of prevention programs contribute to an increase of this occurrence. This study evaluated the sensory-motor and functional response of football athletes of Brasília / DF, after performing the FIFA program "The 11+". 12 male athletes were studied, with mean age of 14 years, height $1.65 \mathrm{~cm}$ and weight $52 \mathrm{~kg}$. There was no control group and break from training. The sensory-motor response was made by EMG in the muscles responsible for kick wide medial, lateral and rectus femoris during the Star Excursion Balance Test, and functionality through the Single Leg Hop Test in both limbs. The standard t-test for dependent samples and the Wilcoxon test for non-normal samples were used. The results showed statistical differences between interventions in EMG of the vastus lateralis $\mathrm{p}=0.02, \mathrm{p}=0.01$ rectus femoris and SLHT the right leg $\mathrm{p}=$ 0.00 . We conclude that the FIFA program "The 11+" promoted increased sensory response-motor and functional in the group of athletes studied.

Keywords: Electromyography. FIFA “The $11+$ +". Prevention.
\end{abstract}




\section{Introdução}

A propriocepção refere-se a qualquer informação postural, posicional ou cinética levada ao Sistema Nervoso Central (SNC) pelos receptores dos músculos, tendões, articulações ou pele (GOETZ, 1999). Para sustentarmos o controle postural e o equilíbrio, dependemos de informações sensoriais periféricas, que têm origem nos nossos sistemas visual, vestibular e somatossensitivo. O sistema nervoso integra essas informações aferentes periféricas para mantermos o controle postural (GOODWIN, 1972). O indivíduo, por meio das informações sensoriais, obtém a percepção do posicionamento e movimentos do corpo, auxiliando-o no controle motor.

Quando um programa de propriocepção é comprometido, negligenciado e realizado de maneira não específica durante as fases do tratamento de reabilitação ou de prevenção, o riscos de recidiva de lesões, comprometendo a integridade dos músculos, ligamentos e cartilagens passam a ser maiores (COHEN; ABDALLA, 2005).

A assimetria entre os membros pode estar associada a um dos fatores que contribuem para a ocorrência de lesão durante a prática esportiva, dentre elas, o futebol, que é reconhecido como um esporte cuja demanda por parte dos jogadores é considerada assimétrica, ou seja, a maioria dos gestos esportivos é realizada pelo membro de prefêrencia do atleta (membro dominante). Entretanto, sobrecarga e compensações que alteram o movimento e a postura. Dessa forma, as diferenças laterais de membros inferiores podem desencadear diversas lesões durante a atividade esportiva, comprometendo o desempenho (COHEN; ABDALLA, 2005).

A eletromiográfia (EMG) é caracterizada por uma técnica de monitoramento da atividade elétrica das membranas excitáveis, representando a medida dos potenciais de ação do sarcolema. O sinal EMG é descrito como a soma álgebra de todos os sinais captados em certa área, podendo ser afetado por diversas estruturas, tais como propriedades anatômicas e fisiológicas (ENOKA, 2000). Dessa forma a eletromiografia se torna uma ferramenta de extrema importância na análise clínica da marcha, fornecendo informações relevantes quanto ao tempo de ativação da musculatura envolvida durante o movimento, intensidade de ativação, variação do ciclo e duração da atividade muscular (ENOKA, 2000).

O futebol é um dos esportes mais praticados em todo o mundo, com cerca de 265 milhões de jogadores masculinos e femininos. Por se tratar de um esporte de contato que desafia a aptidão física e exige uma variedade de habilidades em diversas intensidades, o futebol está associado a um alto risco de lesões nas extremidades inferiores (ALENTORN-GELI et al., 2009; PALLANT, 2005). Schiff et al. (2010) descrevem que as taxas de incidência de lesões no futebol entre jogadores jovens atletas variam entre 2 a 6 lesões por 1000 horas de jogo. Lesões graves no joelho como as lesões do ligamento cruzado anterior, são preocupantes quando falamos em esportes de equipe. Consequentemente, enfatizar a prevenção de lesões no futebol, desenvolver e programar trabalhos de prevenção para jovens jogadores no início de sua carreira se torna uma prática possível e importante para a redução dessas lesões (STEFFEN et al., 2008).

A incidência de lesões no futebol pode ser reduzida por meio de programas preventivos. O Centro de Pesquisa Médica (F-MARC) da Federação Internacional de Futebol (FIFA) desenvolveu um programa de prevenção que consiste em exercícios simples e de fácil memorização, que, quando realizados corretamente, promovem aumento significativo no equilíbrio central, no treinamento excêntrico da musculatura, no treinamento proprioceptivo e na pliometria do atleta (DVORAK, 2005). Kirkendall et al. (2010) relatam que um programa de prevenção tem sido eficaz na prevenção de lesões comuns no futebol.

O objetivo desse estudo foi avaliar a resposta sensório-motora por meio da atividade eletromiográfica dos músculos vasto medial, vasto lateral e reto femoral durante a execução do Star Exercusion Balance Test e a resposta funcional por meio do Teste funcional de salto Single Leg Hop Test.

\section{Metodologia}

\subsection{Voluntários}

Foram avaliados 12 atletas da categoria de base de futebol na cidade de Brasília-DF, onde todos eram do gênero masculino e apenas um canhoto. A idade média dos atletas foi de 14 anos (DP de 0,49), a média da estatura 1,65 (DP 0,09 ) e o peso médio aproximadamente $51,33 \mathrm{Kg}$ (DP $9,00)$. Todos os participantes não apresentavam histórico de lesão em membros inferiores. O estudo foi aprovado pelo Comitê de Ética do Centro Universitário de Brasília - UniCEUB, sob o código CAAE: 08925812.0.0000.0023. Os participantes foram recrutados de forma vo- 
luntária pelo pesquisador responsável atletas da categoria de base de uma escola de futebol de Brasília-DF, durante o período de treinamento.

\subsection{Materiais}

Foi utilizado o eletromiógrafo de superfície validado da marca EMG System do Brasil ${ }^{\circledR}$ com 8 canais (VASCONCELOS, 2005), Bolas da marca penalty e cones da marca Kpista ${ }^{\circ}$.

\subsection{Procedimentos}

Inicialmente os voluntários e os responsáveis pelos atletas foram orientados a assinar o termo de consentimento livre e esclarecido e a ficha de anamnese para a identificação dos participantes da pesquisa.

Após tomarem consentimento sobre os procedimentos do estudo, realizou-se avaliação inicial (pré-intervenção) dos atletas, com o intuito de obter os dados da resposta sensório-motora por meio da EMG de superfície durante a execução do Star Excusion Balance Test e a resposta funcional por meio do Single Leg Hop Test antes da aplicação do programa preventivo. Posteriormente, a avaliação inicial foi aplicado o programa de prevenção de lesões validado pela F-MARC/FIFA The 11+ (DVORAK, 2005), realizado duas vezes por semana (Terças e Quintas) por um período de 4 semanas consecutivos em uma escolinha de futebol de base de Brasília-DF. Após esse período de intervenção, uma avaliação final (pós-intervenção) foi realizada utilizando os mesmo procedimentos da avaliação inicial, com o intuito de obtermos dados após a aplicação do programa preventivo. Todos os procedimentos foram realizados pelo responsável da pesquisa e todos os atletas mantiveram seus treinamentos de 3 vezes por semana normais.

Foram realizados dois procedimentos de avaliação, um para a obtenção da resposta sensório-motora e um para a resposta funcional dos atletas, por meio da Eletromiografia de superfície e do Teste funcional de salto Single Leg Hop Test respectivamente.

\subsection{Resposta Sensório-Motora}

Foi feita a assepsia da região anterior da coxa dos membros do voluntário com álcool 70\%, e quando necessário, a tricotomização do local. Feito isso, eram marcados os pontos para aquisição da ativação muscular e, posteriormente, posicionados os eletrodos com dois centímetros de distância intereletrodo (SENIAM, 2013). Para a aquisição dos sinais, a coleta foi realizada em laboratório específico, a fim de reduzir a interferência de ruídos.
O procedimento de conexão dos eletrodos foi realizado com os canais: canal 3: Reto femoral; Canal 4: Vasto Lateral; Canal 6: Vasto Medial; e o eletrodo de referência localizado no maléolo medial do membro a ser avaliado.

O procedimento da aquisição da resposta sensório-motora foi realizado na semana subsequente ao termino da aplicação do programa de prevenção adotado no estudo. Após a explicação ao voluntário, o teste se iniciava assim que ele estivesse preparado. Com o objetivo de termos o maior tempo de captação da atividade elétrica muscular, a resposta sensório-motora foi obtida por meio da EMG de superfície durante a realização do teste de estrela Star Excursion Balance Test, em que o atleta foi orientado a realizar o teste por um período de 60 segundos.

\subsection{Funcionalidade}

Projetado para avaliar o desempenho funcional, a resposta funcional dos atletas foi obtida por meio do teste funcional de salto Single Leg Hop Test, com o intuito de imitar as exigências de aptidão física do futebol.

Inicialmente, foi colocada ao chão uma fita crepe com uma distancia de 3 metros e marcados dois pontos iniciais fixos, a fim de delimitar o inicio dos saltos para a perna direita e esquerda.

O procedimento do estudo foi explicado ao voluntário e o teste se iniciava assim que ele estivesse preparado. $\mathrm{O}$ teste consistia em realizar três saltos com apenas uma perna fixa no chão na maior distancia possível. Não houve restrições quanto à ajuda de membros superiores durante a execução do salto, foi orientado ao voluntario que permanecesse no local após o salto para a realização da medida com uma fita antropométrica. A média dos três saltos foi utilizada para fins estatísticos.

\subsection{Análise estatística}

Para testar a normalidade dos dados, foi realizado o teste de Kolmogorov - Smirnov, com nível de confiança de $95 \%$. Foi considerado normal a variável que apresenta $\mathrm{p}$-valor $>0,05$. Apenas as variáveis: músculo reto femoral perna direita pré- intervenção, músculo reto femoral perna direita pós-intervenção e músculo vasto medial perna esquerda pré-intervenção, não seguiram a distribuição de probabilidade normal, pois p-valor calculado é $<0,05$. O teste T pareado foi utilizado, pois existiram duas condições experimentais, em 
que os mesmos participantes tomaram parte em ambas as condições, com dados obtidos de populações normalmente distribuídas. Para as variáveis que não apresentaram distribuição de probabilidade normal, foi utilizado o teste não paramétrico de Wilcoxon, similar ao teste t-Student pareado para dados não normais, com o intuito de comparar duas populações relacionadas (antes e após intervenção). Valores menores que 0,05 foram considerados estatisticamente significativos.

\section{Resultados}

Os quadros 1 e 2 mostram os valores que representam a distribuição da Root Mean Square ( $R$ MS) nos músculos reto femoral, vasto lateral e vasto medial durante o período pré e pós-intervenção.

Quadro 1 - Distribuição da RMS para os músculos da perna direita

\begin{tabular}{|l|c|c|c|c|c|}
\hline $\begin{array}{c}\text { Músculos- } \\
\text { Membro } \\
\text { Direito }\end{array}$ & $\begin{array}{c}\text { RMS pré- } \\
\text { intervenção }\end{array}$ & $\begin{array}{r}\text { Desvio } \\
\text { padrão }\end{array}$ & $\begin{array}{c}\text { RMS pós- } \\
\text { intervenção }\end{array}$ & $\begin{array}{c}\text { Desvio } \\
\text { padrão }\end{array}$ & p-valor \\
\hline $\begin{array}{l}\text { Reto } \\
\text { femoral }\end{array}$ & 128,24 & 100,69 & 133,34 & 102,57 & $0,02^{*}$ \\
\hline $\begin{array}{l}\text { Vasto } \\
\text { lateral }\end{array}$ & 130,30 & 45,05 & 134,07 & 44,33 & $0.01^{*}$ \\
\hline $\begin{array}{l}\text { Vasto } \\
\text { medial }\end{array}$ & 166,25 & 20,27 & 170,18 & 20,35 & 0,30 \\
\hline
\end{tabular}

Quadro 2 - Distribuição da RMS para os músculos da perna esquerda

\begin{tabular}{|l|c|c|c|c|c|}
\hline $\begin{array}{l}\text { Músculos } \\
\text { esquerdo }\end{array}$ & $\begin{array}{c}\text { RMS pré- } \\
\text { intervenção }\end{array}$ & $\begin{array}{l}\text { Desvio } \\
\text { padrão }\end{array}$ & $\begin{array}{c}\text { RMS pós } \\
\text { intervenção }\end{array}$ & $\begin{array}{c}\text { Desvio } \\
\text { padrão }\end{array}$ & p-valor \\
\hline $\begin{array}{l}\text { Reto } \\
\text { femoral }\end{array}$ & 68,58 & 21,14 & 69,55 & 20,99 & 0,18 \\
\hline $\begin{array}{l}\text { Vasto } \\
\text { lateral }\end{array}$ & 129,20 & 41,17 & 130,44 & 40,34 & 0,61 \\
\hline $\begin{array}{l}\text { Vasto } \\
\text { medial }\end{array}$ & 144,09 & 45,60 & 147,15 & 42,07 & 0,24 \\
\hline
\end{tabular}

O Quadro 3 mostra os valores que representam a média dos saltos realizados durante o Single Leg Hop Test.
Quadro 3 - Distribuição da média de saltos do teste Single Leg Hop Test

\begin{tabular}{|l|c|c|c|c|c|}
\hline & $\begin{array}{l}\text { Média pré- } \\
\text { intervenção }\end{array}$ & $\begin{array}{l}\text { Desvio } \\
\text { padrão }\end{array}$ & $\begin{array}{l}\text { Media pós- } \\
\text { intervenção }\end{array}$ & $\begin{array}{l}\text { Desvio } \\
\text { padrão }\end{array}$ & p-valor \\
\hline $\begin{array}{l}\text { SLHT- Perna } \\
\text { direita }\end{array}$ & $1,59 \mathrm{~cm}$ & 0,14 & 1,67 & 0,15 & $0,00^{*}$ \\
\hline $\begin{array}{l}\text { SLHT- Perna } \\
\text { Esquerda }\end{array}$ & 1,62 & 0,21 & 1,63 & 0,21 & 0,36 \\
\hline
\end{tabular}

Pode-se observar que, nos quadros 1 e 2, houve diferenças significativas entre a ativação elétrica muscular pré e pós-intervenção para os músculos reto femoral e vasto lateral do membro inferior. O Quadro 3 mostra diferença estatisticamente significativa entre a resposta funcional do membro inferior direito pré e pós-intervenção. Ao aplicar os dados obtidos nas avaliações ao tratamento estatístico, pode-se observar que existe diferença significativa entre o músculo reto femoral, vasto lateral durante a captação eletromiográfica no SEBT e diferença no teste SLHT perna direita pré-intervenção e pós-intervenção. O p-valor para essas variáveis é menor que 0,05, representando diferença significativa entre as médias antes e após a intervenção e aumento de 2,89\%,3,98\% e 4,87\% na média dos músculos reto femoral, vasto lateral e o teste SLHT perna direita após a intervenção, respectivamente. $\mathrm{O}$ que nos permite afirmar que foram encontradas diferenças significativas entre a ativação muscular e funcionalidade dos atletas estudados para o membro direito após a aplicação do programa de prevenção FIFA The 11+, dando-nos o feedback a respeito da utilização do membro dominante na pratica do futebol.

\section{Discussão}

Conforme os resultados encontrados no presente estudo, e o feedback proporcionado acerca da influencia do membro dominante nos resultados, podemos correlacionar com estudos que demonstraram a utilização do membro dominante na pratica do futebol. Alter (1999) destacou que a preferência lateral, relacionada à dominância para o uso de um dos membros constitui característica humana descrita como o favorecimento de um dos lados do corpo para a realização de atividade esportiva. Kramer e Balsor (1990) relataram que, nas atividades esportivas, o membro dominante pode apresentar várias diferenças funcionais quando comparado ao membro não dominante. 
Dentre as atividades esportivas, o futebol é reconhecido como um esporte cuja demanda é assimétrica, na qual a maioria dos gestos esportivos é executada por membros preferidos (NELSON, 2000). A prevenção das lesões no esporte pode ser possível por meio da determinação dos fatores de risco de uma lesão pré-determinada. KIANI et al. (2010) relata que o programa FIFA 11+ é um programa de prevenção multifuncional específico para o futebol, em que incluem a estabilidade do centro do corpo, equilíbrio e controle neuromuscular que promovem padrões de movimentos adequados e destinados a diminuir as lesões corporais.

Estudos avaliaram a força e o recrutamento das unidades motoras dos músculos extensores do joelho após o programa preventivo FIFA the 11+. Alguns dados observados podem se correlacionar de certa forma com os resultados encontrados no presente estudo. BRITO et al. (2010) demonstraram que o FIFA 11+ melhorou a força e o aumento da ativação dos músculos extensores do joelho a $60^{\circ} \mathrm{e} 180^{\circ}$ na perna dominante dos jogadores de futebol do sexo masculino não profissionais, o que corroborou com os achados de Daneshjoo (2013) que, em relação ao grupo analisado, revelou que o programa de aquecimento aumenta a força e o recrutamento muscular concêntrico do quadríceps em várias velocidades do teste isocinético, aumentando assim, a força do quadríceps após o FIFA11+ apenas na perna dominante.

Tais dados podem se correlacionar com nossos achados, devido ao fato de a eletromiografia de superfície apresentar feedback da ativação e recrutamento de unidades motoras musculares. Tal fato, se correlaciona com as variáveis apresentadas no estudo, devido ao fato de estarem extremamente proporcionais e, quanto maior o aumento no número de unidades motoras recrutadas durante determinada ação, maior a força implementada, o que pode ser mais evidenciada nas velocidades mais baixas no teste isocinético (DANESHJOO, 2013).

O músculo do quadríceps é mais ativo durante a fase do pouso, desacelerações e durante a fase de decolagem e frenagem do salto, fato que pode corroborar com o resultado deste estudo, em que foi encontrada diferença significativa para o membro direito, quando comparado pré e pós intervenção para a avaliação funcional utilizando o teste funcional de salto SLHT (DANESHJOO, 2013).

Os exercícios preventivos THE 11+ incorporam um ou mais componentes de exercícios que incluem a pliometria, equilíbrio, propriocepção e força com pa- drões de movimento, a fim de evitar lesões em jogadores de futebol. Em uma meta-análise, Hewett et al. (2006) demonstraram que o treinamento e aquecimento pliometrico foi um componente que reduziu eficazmente taxas de lesão e que o treinamento de pliometria induz produção e adaptações neuromusculares maiores de força e recrutamento de unidades motoras nos músculos em membros dominantes.

Bizzini, Junge e Dvorak (2007) relataram que o FIFA The 11+ permite a prevenção de lesões e, também, consequentemente melhoram o rendimento físico do atleta. Esses autores observaram em estudo com jogadores italianos que após a adição do programa de prevenção de lesões em seu calendário habitual, os mesmos apresentaram, depois de três meses, melhora na força, equilibrio, agilidade e controle neuromuscular, comparado a outros jogadores que fizeram a temporada habitual convencional.

Bizzini, Junge e Dvorak (2007) em um estudo semelhante a este aplicaram o programa de exercícios preventivos de lesões FIFA The 11+, com duas mil jovens jogadoras de diversas equipes do mundo pelo menos duas vezes por semana, em que as atletas estudadas apresentaram redução no número de lesões de $30 \%$ a $50 \%$ em comparação com as equipes que fizeram o aquecimento usual FIFA THE 11+. No estudo apresentado neste artigo identificamos aumento na resposta da atividade elétrica muscular e na resposta funcional do membro dominante dos atletas. Esse estudo limitou-se ao fato de a literatura não abordar estudos em que associem a utilização da eletromiografia à resposta e ao feedbak acerca da ativação muscular em determinados exercícios durante a fase de reabilitação ou prevenção.

Recomenda-se que novos estudos sejam realizados, a fim de comparar a utilização de programas preventivos com outros treinamentos proprioceptivos para oferecer bases científicas aos fisioterapeutas do esporte para um adequado método de programas preventivos e de reabilitação envolvendo o atleta.

\section{Conclusão}

Os resultados do presente estudo possibilitaram concluir que a resposta da atividade elétrica e sensório-motora dos músculos vasto medial, vasto lateral e reto femoral, juntamente com o teste funcional de salto SLHT para o membro direito, apresentaram ganho de resulta- 
dos estatisticamente significativos após a aplicação do programa de exercícios de aquecimento FIFA The 11+.

\section{Referências}

ALENTORN-GELI, E. et al. Prevention of non-contact anterior cruciate ligament injuries in soccer players. Part 1: mechanisms of injury and underlying risk factors. Knee Surgery, Sports Traumatology, Arthroscopy, Heidelberg, v. 17, n. 7, p. 705-729, Jul. 2009. doi: 10.1007/ s00167-009-0813-1.

ALTER, M. J. Ciência da flexibilidade. Porto Alegre: Artmed, 1999.

BIZZINI, M.; JUNGE, A.; DVORAK, J. A complete warm-up programme to prevent injuries. Basel: FIFA, 2007. Disponível em: <http://www.f-marc.com/downloads/workbook/11plus_workbook_e.pdf>. Acesso em: 10 set. 2015.

BRITO, J. et al. Isokinetic strength effects of FIFA's “The $11+$ " injury prevention training programme. Isokinetics and Exercise Science, Amsterdam, v. 18, n. 4, p. 211-215, Oct./Dec. 2010. doi: 10.3233/IES-2010-0386.

COHEN, M.; ABDALLA, R. J. Lesões nos esportes: diagnóstico, prevenção, tratamento. Rio de Janeiro: Revinter, 2005.

CORAZZA, S. T.; PEREIRA, E. F.; VILLIS, J. M. C. Propriocepção e a familiarização ao meio líquido. Educación Física y Deportes, Revista Digital, Bueno Aires, ano 10, n. 82, Mar. 2005.

DANESHJOO, A.; RAHNAMA, N.; MOKHTAR, T. Effectiveness of injury prevention programs on developing quadriceps and hamstrings strength of young male professional soccer players. Journal of Human Kinetics, Kraków, v. 39, p. 115-125, Dec. 2013. doi: 10.2478/ hukin-2013-0074.

EKLUND, G. Position sense and state of contraction: the effects of vibration. Journal of Neurology, Neurosurgery and Psychiatry, London, v. 35, n. 5, p. 606-611, Oct. 1972.

ENOKA, R. M. Bases neuromecânicas da cinesiologia. São Paulo: Manole, 2000.
GOETZ, C. G. Textbook of clinical neurology. Philadelphia: W. B. Saunders, 1999.

GOODWIN, G. M.; MCCLOSKEY, D. I.; MATTHEWS, P. B. C. The contribution of muscle afferents to kinesthesia shown by vibration induced illusions of movement and by the effects of paralyzing joint afferents. Brain, Oxford, v. 95, n. 4, p. 705-748, Jan. 1972. doi: 10.1093/ brain/95.4.705.

HART, S.; GABBARD, C. Examining the stabilizing characteristics of footedness. Laterality: Asymmetries of Body, Brain and Cognition, London, v. 2, n. 1, p. 17-26, Sept. 1997. doi: 10.1080/713754251.

HEALEY, J. M.; LIEDERMAN, J.; GESCHWIND, N. Handedness is not a unidimensional trait. Cortex, Milan, v. 22, n. 1, p. 33-53, Mar. 1986. doi: 10.1016/S00109452(86)80031-4.

HERTEL, J.; MILLER, S. J.; DENEGAR, C. R. Intratester and intertester reliability during the star excursion balance tests. Journal of Sport Rehabilitation, Champaign, v. 9, n. 2, p. 104-116, May 2000.

HEWETT, T., FORD, K. R.; MYER, G. D. Anterior cruciate ligament injuries in female athletes Part 2, A meta-analysis of neuromuscular interventions aimed at injury prevention. The American Journal of Sports Medicine, Baltimore, v. 34, n. 3, p. 490-498, Mar. 2006. doi:10.1177/0363546505282619.

HOLLER, A. A.; ANDRÉ, E. S. Efeitos do laser de baixa intensidade HeNe sobre a atividade eletrográfica do músculo gastrocnêmio de ratos Wistar submetidos à lesão do nervo ciático. Fisioterapia Brasil, Rio de Janeiro, v. 8, n. 2, p. 89-98, Mar./Abr. 2007.

JUNGE A. et al. Football injuries during FIFA tournaments and the Olympic Games, development and implementation of an injury-reporting system. The American Journal of Sports Medicine, Baltimore, v. 32, n. Suppl 1, p. 80S-89S, Jan./Fev. 2004. doi:10.1177/0363546503261245.

JUNGE, A.; DVORAK, J. Injuries in female football players in top-level international tournaments. British Journal of Sports Medicine, London, v. 41, Suppl. 1, p. i3-i7, Aug. 2007. doi: 10.1136/bjsm.2007.036020. 
KIANI, A. et al. Prevention of soccer-related knee injuries in teenaged girls. Archives of Internal Medicine, Chicago, v. 170, n. 1, p. 43-49, Jan. 2010. doi: 10.1001/archinternmed.2009.289.

KRAMER, J. F.; BALSOR, B. E. Lower extremity preference and knee extensor torques in intercollegiate soccer players. Canadian Journal of Sport Sciences, Champaign, v. 15, n. 3, p. 180-184, Sept. 1990.

LENT, R. Cem bilhões de neurônios: conceitos fundamentais de neurociência. São Paulo: Atheneu, 2004.

PALLANT, J. F. SPSS survival manual: a step by step guide to data analysis using SPSS for windows. Crows Nest: Allen \& Unwin, 2005.
SENIAM. Recommendations for sensor locations in hip or upper leg muscles. Disponível em: <http://seniam.org/leg_location.htm>. Acesso em: 18 jun. 2013.

SCHIFF M. A. et al. Soccer injuries in female youth players: comparison of injury surveillance by certified athletic trainers and internet. Journal of Athletic Training, Dallas, v. 45, n. 3, p. 238-242, May/Jun. 2010. doi: 10.4085/1062-6050-45.3.238.

STEFFEN, K. et al. Preventing injuries in female youth football-a cluster-randomized controlled trial. Scandinavian Journal of Medicine and Science in Sports, Copenhagen, v. 18, n. 5, p. 605-614, Oct. 2008. doi: 10.1111/j.1600-0838.2007.00703.x. 